\title{
Deep Processing of Rice and Comprehensive Utilization of Its By-products
}

\author{
Wen $\mathrm{Li}^{1, \#}$, Yu-Shui Ma ${ }^{1,2, \#}$, Qin-Lu Lin ${ }^{1, *}$, Da Fu ${ }^{1,2, *}$ \\ ${ }^{1}$ National Engineering Laboratory for Rice and By-product Deep Processing, College of Food Science and Engineering, \\ Central South University of Forestry and Technology, Changsha 410004, China \\ ${ }^{2}$ Central Laboratory for Medical Research, Shanghai Tenth People's Hospital, \\ Tongji University School of Medicine, Shanghai 200072, China \\ ${ }^{\#}$ These authors contributed equally to this work. \\ *Corresponding author: linq10403@126.com, fu800da900@126.com
}

Received February 11, 2019; Revised March 21, 2019; Accepted April 27, 2019

\begin{abstract}
China is a big country in the production and consumption of rice in the world. However, for a long time, China's rice processing is only in the primary processing state that meets people's demand for rations, and the level of comprehensive utilization of deep processing and its by-products is low. This paper reviews the main raw and byproducts of rice, such as rice protein, rice starch and by-products of rice processing (including broken rice, rice bran and rice husk), aiming to further improve the comprehensive utilization of rice resources and increase the added value of rice and its by-products to create more economic and social benefits. The vigorously developing and promoting the deep processing technology of rice and its by-products will extend the rice industry chain and greatly increasing the added value of rice, make full use of resources to supplement the shortage of high-quality edible oil, protein, starch and other products.
\end{abstract}

Keywords: rice, by-product, deep processing, comprehensive utilization

Cite This Article: Wen Li, Yu-Shui Ma, Qin-Lu Lin, and Da Fu, "Deep Processing of Rice and Comprehensive Utilization of Its By-products." Journal of Food and Nutrition Research, vol. 7, no. 5 (2019): 370-376. doi: 10.12691/jfnr-7-5-6.

\section{Introduction}

China is a big agricultural country. Rice is the largest grain crop with the largest total output in China. It has always dominated grain production and consumption [1]. However, China's rice processing is currently only in a state of initial processing that meets the demand for ration rice. The effective utilization rate is only $60-65 \%$, deep processing only accounts for $20 \%$, and the comprehensive utilization level of by-products is low, which far behind developed countries [2]. Efficient value-added deep processing of japonica rice and by-products with poor quality of food, and ultimately industrialization, is an effective measure to transform from a large rice producing country to a rice producing power [3].

Rice deep processing is based on rice, brown rice, rice germ, etc., and is processed into various products by physical, chemical, biochemical and other technologies [4]. In recent years, with the application of biotechnology (including enzyme technology, fermentation technology, etc.), membrane separation technology, supercritical extraction technology, ultrafine pulverization technology and microcapsule technology in the development of grain, oil and food, it provides for deep processing of rice with modern means of production [5].
These high-tech not only ensure the nutrition, safety, hygiene and flavor of rice deep-processing products, but also make comprehensive utilization of rice resources, so as to obtain good social and economic benefits [6].

\section{Rice Modified Starch}

Starch is the main component of rice, rice starch granules are very small (between 3-8 $\mu \mathrm{m}$ ) and the particle size is uniform. Rice starch is high-purity rice starch (lower protein content) on the international market due to its unique properties [7]. The demand is large, so it has great market prospects. At present, Europe and the United States have set off a wave of research and development of rice starch. Using modern biotechnology, rice starch can be modified into more distinctive and new-purpose products such as resistant starch, porous starch, slowly digested starch, and new fat substitute.

\subsection{Slowly Digesting Starch}

Slowly digesting starch is a starch that can be slowly and completely degraded by enzymes. Its slow digestion in the human small intestine is very beneficial to human health. It can not only improve sugar load as a new food for diabetic patients, but also exercise for athletes. The 
process towel provides a stable and long-lasting energy release beam to maintain endurance and is therefore receiving more and more attention. At present, the preparation of rice slowly digested starch mainly includes enzymatic and physical methods.

Chen Lei et al. [8] used the pullulanase debranching treatment and heat treatment to prepare rice slowly digested starch. The results showed that under the optimal conditions, the slow-digested starch content obtained by the pullulan debranching treatment was $50.1 \%$, which was significantly higher than that of the slow-digested rice starch prepared by heat treatment (content $7.9 \%$ ).

Zhang et al. [9] bathed the waxy rice starch for 30 minutes, and then cooled to 25 degrees, sealed for 4 hours at 4 degrees, and then stored at 25 degrees for 24 hours. After 7 days of storage in this way, the slowly digested starch content in the waxy rice starch can reach $51.62 \%$.

The United States has developed a new rice starch product, Ricemic, which uses rice flour as a raw material to separate proteins, which are then processed into $100 \%$ delayed digestion, $50 \%$ accelerated digestion and 50\% delayed digestion by heat and enzyme treatment. Such modified rice starch has been clinically proven to improve sugar load, which will become a new food for diabetic patients [10]. Because this slowly digested starch allows athletes to have a consistent and long-lasting energy release during exercise to maintain endurance, the product can also be used as a carbohydrate supplement for athletes, especially marathon runners.

\subsection{Resistant Starch}

Resistant starch refers to a general term for starch and its degradation products that are not absorbed by the small intestine of healthy humans. Resistant starch has a similar effect as dietary fiber, has no energy, and has physiological functions such as preventing diabetes, protecting the intestine, improving blood lipids, and controlling body weight [11]. Resistant starch is mainly used in medium and low moisture foods.

Granule resistant starch provides better appearance, texture and mouth feel than traditional fibers, improving the swelling and brittleness of food. Resistant starch is mainly formed by aging of amylose, so that the structure of the starch granules can be destroyed under high temperature and hot and humid conditions, the starch is fully gelatinized, and then the resistant starch can be prepared by taking measures to maximize the aging of the starch.

At present, methods for preparing rice-resistant starch mainly include an acid-denatured boiling water bath method and a pressure heat treatment method [12]. Resistant starch is not easily digested (even if it does not extend the digestion time) and is suitable for obese and diabetic patients. Unlike ordinary fiber components, it absorbs a lot of water. When added to low-moisture products, it does not affect its taste and does not change the flavor of food. It can be used as a low-calorie food additive.

\subsection{Porous Starch}

Porous starch refers to a honeycomb porous starch carrier formed by enzymatically treating native starch.
Porous starch has many pores extending to the center of the starch granules on its surface, and the center of the starch granules is hollow, so it has good adsorption properties and can be used as an adsorption carrier for functional substances (such as pharmaceuticals, perfumes, pigments, health care substances).

Porous starch is widely used in the pharmaceutical, chemical and food industries [13]. The rice starch has small particle size and large specific surface area, so the prepared porous starch has stronger adsorption capacity than other kinds of starch. At present, the preparation method of rice porous starch has a single enzymatic hydrolysis or enzymatic hydrolysis and cross-linking, esterification and the like [14]. Crosslinking can increase the mechanical strength and swell resistance of porous starch, and esterification can increase the hydrophobicity of porous starch, thereby increasing its ability to adsorb non-polar and weakly polar substances. Sheng et al. used alpha-amylase to prepare glutinous rice porous starch, which improved the ability of glutinous rice starch to absorb liquid [15]. Tao et al. prepared rice porous starch with alpha-amylase and glucoamylase with a ratio of 1:12. The water absorption and oil absorption rates were $125.8 \%$ and $163.2 \%$, respectively [16]. Srikaeo et al. [17] prepared cross-linked esterified porous rice starch by cross-linking phosphorus oxychloride, complex enzyme hydrolysis and octenyl succinate, and its adsorption capacity for methylene blue and cross-linked porous rice starch and porous rice starch. Their results showed that the cross-linked esterified porous rice starch had the strongest adsorption capacity, the cross-linked porous rice starch was the second, and the porous rice starch was the weakest.

\subsection{Starch-based Fat Substitute}

Rice starch to make fat substitute technology is a hightech application of biotechnology to convert rice starch into oil-free fat. The rice starch fat substitute mainly includes modified or modified rice starch, ultrafine powder, and low devalue value maltodextrin [18]. Starch fat substitutes mimic the texture and mouthfeel of fat. The mechanism is that internal amylose and amylopectin work together to form a gel. The three-dimensional network structure of the gel can retain a part of water, and the trapped water has a certain fluidity, and can produce a cream-like effect under the action of the oral cavity. The fat substitute based on rice starch has different properties from other starch raw materials because the rice flour particles are small and close to the size of the homogenized fat globules, which is suitable for use as a fat substitute, and the rice starch hydrates to form [19].

The system is softer and more delicate, and it can simulate the taste of fat. The new fat substitute has a creamy appearance and a mouth feel. It is very suitable for processing yoghurt and some dairy products that replace cream. It can be processed into a hydrogenated fat for margarine production. A\&B Ingredient, the world's largest rice starch producer, has used modified rice starch for the production of cream-free cheese, low-fat ice cream, fat-free margarine, sauce and salad dressing, with considerable economic benefits [20]. 


\section{Rice Protein and Its Modification Technology}

The protein content in rice is about $8 \%$, and the protein content of rice slag, which is a by-product of the production of starch sugar by using early indica rice or broken rice as raw material, is up to $50 \%$, which is a high-quality raw material for extracting rice protein [21]. Rice protein has received wide attention from the world because of its high bioavailability and low allergic properties. Rice protein is a high-quality protein, which is manifested in the following three aspects: 1) the amino acid composition of rice protein is reasonable, which is very close to the ideal model recommended by World Health Organization (WHO) the Food and Agriculture Organization of the United Nations (FAO) [22]. Its bio-price is 77, which higher than other food crops; 2) rice protein is a hypoallergenic protein that is safer and more reliable than other food crops. It can be used as a base for the development of infant foods; 3) rice protein isolates regulate blood pressure, lower cholesterol, and reduce atherosclerosis [23].

However, since gluten, which accounts for about $80 \%$ of rice protein, has high hydrophobicity, the solubility of rice protein is poor, and the functional properties such as emulsifying property, gelation property, and foaming property are unsatisfactory, which limits its applications in the food field [24]. Therefore, rice protein modification has become a hot topic in current research, and the modification can improve the functional properties of rice protein, thereby expanding its application in food and other fields.

Rice protein can be classified into four categories according to Osborne's solubility-based classification: albumin, globulin, prolamin and gluten [25]. Using hypoallergenic and highly nutritious rice protein, it can develop high-protein hypoallergenic infant formula for infant-sensitive diarrhea; use edible rice protein concentrate and pullulan to make edible film. The concentrate of rice gluten can be used for aquatic feed. The insolubility of gluten keeps the water quality clear, and good digestion can reduce the defecation of aquatic animals and reduce water pollution. Enzymatic hydrolysis of rice protein can produce high-protein nutritious rice flour with protein content of more than $80 \%$, used in baby food, baked goods, breakfast food, snack food, etc [26]. In addition, rice protein is used as a resistance protein, and as a carrier of some drugs, it will play its unique role in medical and veterinary medicine [27].

\section{Broken Rice}

Based on the existing rice milling technology, 10-15\% of broken rice will inevitably be produced during the processing of rice. The nutrients of broken rice are similar to rice, but the price is only $1 / 3$ to $1 / 2$ of rice [28]. According to rough estimates, the amount of broken rice produced each year is nearly 100 million tons, which causes great waste of rice resources and seriously affects the economic benefits of polished rice processing [29]. Therefore, research and development of broken rice products is becoming more and more urgent.

The comprehensive utilization of broken rice is mainly in the following two aspects: First, the development and utilization of higher content of starch in broken rice. At present, the new products produced by broken rice starch in China mainly include fructose syrup, maltitol and maltodextrin powder, sorbitol, liquid wine, beverages, etc. [30]; the second is to use the protein in the broken rice, the high protein rice powder obtained by increasing the protein content in the broken rice, can be used as a high protein food for infants, the elderly and patients [31]. The rice residue after the use of broken rice starch contains more protein and can be used as a product for producing soy sauce, foaming powder, protein feed, peptone and yeast medium [32].

\section{Comprehensive Utilization of Rice Bran}

Rice bran is a surface layer attached to brown rice after shelling, consisting of peel, seed coat, exoemulsion and aleurone layer. Although rice bran only accounts for $6 \%$ to $8 \%$ of rice quality, it contains $60 \%$ to $70 \%$ of physiologically active ingredients in rice, and more than $90 \%$ of essential elements [33]. Its chemical composition is sugar and fat (14-24\%). ) and protein (12-16\%), and also contains more vitamins, phytate and mineral nutrients, and contains nearly 100 kinds of tocopherol, tocotrienol, oryzanol, octadecyl alcohol A biologically active substance with various functions, which has various functions such as preventing cardiovascular diseases, regulating blood sugar, losing weight, preventing tumors, anti-fatigue and beauty $[34,35,36,37]$. As the most important by-product of rice processing, rice bran has long been used mainly as animal feed, and it has not been fully utilized, resulting in serious waste of resources. The comprehensive utilization of rice bran resources is an effective means to increase the economic benefits of rice processing enterprises, and has become a research hotspot in recent years.

\subsection{Rice Bran Oil}

Rice bran oil is a nutrient-rich vegetable oil, in which saturated fatty acids account for $15 \%$ to $20 \%$, unsaturated fatty acid content is over $80 \%$, linoleic acid content is about $38 \%$, and oleic acid content is about $42 \%$, which is in line with the recommendations of the International Health Organization [38]. Rice bran oil has the scent of aroma, high temperature frying, long-term storage and almost no harmful substances, which is unmatched by any kind of vegetable oil. Because of the superior performance of rice bran oil, it has become another new health food oil after sunflower oil and corn germ oil. Rice bran oil not only has a complete and reasonable fatty acid composition, but also contains a variety of physiologically active ingredients such as Vitamin E, oryzanol, phytosterols, and squalene [39]. These substances can effectively lower the concentration of low-density cholesterol in the blood, increase the concentration of high-density cholesterol, and prevent cardiovascular diseases, regulate blood sugar, and improve atherosclerosis [40]. At present, the extraction of rice bran oil mainly includes pressing method, microwave assisting method, and leaching method [41]. Among them, the pressing method is most commonly used, but the obtained rice bran oil contains more impurities and still needs further refining. 


\subsection{Oryzanol}

Oryzanol inhibits the absorption and synthesis of cholesterol, promotes the metabolism and excretion of cholesterol, and has the effects of lowering blood fat and preventing cardiovascular diseases such as atherosclerosis [42]. In China, oryzanol has been used as a medicine, and Japan has applied oryzanol to food for more than 20 years. As a by-product of the production of rice bran oil, the extraction of oryzanol is generally combined with the refining of butter oil. In recent years, oryzanol's production methods have been continuously improved in terms of simplifying processes, increasing yield, and reducing costs. According to reports, under optimal conditions, the yield of oryzanol can be doubled compared to the traditional weak acid substitution method.

\subsection{Rice Bran Wax}

Rice bran wax is mainly composed of higher fatty alcohol esters. The esters can be decomposed and converted to obtain biologically active functional substances, 28 alkanols and 30 alkanols, which are U. S. Food and Drug Administration (FDA)-approved functional additives, which can improve mobile endurance and regulate fat metabolism [43]. And lowering cholesterol, it can be widely used in functional foods, various nutritional supplements, medicines, cosmetics and high-grade feeds, and its market share is also expanding [44]. Functional foods with 28 alkanols as the main ingredients are popular in the United States, Japan and other countries. The market prospects are very broad and the economic benefits are also very significant.

\subsection{Rice Bran Nutrients and Nutrient Fiber}

Rice bran nutrients and nutrient fiber are nutrients that the body needs very much but are difficult to ingest in daily diet. Rice bran nutrients and nutrient fiber not only contain high-quality protein and unsaturated fatty acids, but also contain vitamins and minerals and various physiologically active ingredients required by the human body every day [45]. Using the rich and comprehensive nutrients and dietary fiber of rice bran to develop nutritious and healthy foods, it is the earliest and most rice bran food. At the same time, rice bran is also rich in protein, vitamins and dietary fiber to produce high-nutrition foods and health products, such as rice bran bread, rice bran biscuits, rice bran noodles and other foods. In addition, the solid beverage produced from rice bran nutrients and rice bran nutrition fiber has a rich cocoa flavor and a favorite rich moisturizing taste. The eater supplements the daily protein, unsaturated fatty acids, vitamins, minerals, etc., and can also supplement the dietary fiber to obtain a smooth and laxative health care function [46]. The rice bran nutrient solid beverage and the rice bran nutrient fiber solid beverage have pure natural and low price. Such advantages, and therefore have a strong competitive advantage and market potential.

\subsection{Rice Bran Polysaccharide}

Rice bran polysaccharide is mainly found in the rice peony skin layer, and is complex with cellulose, hemicellulose and other components. It is a complex heteropolysaccharide. Unlike starch polysaccharides, rice bran polysaccharides are mainly composed of xylose, mannose, rhamnose, galactose, arabinose and glucose. The content of impurities in the crude rice bran polysaccharide is high. To obtain the uniform polysaccharide, further separation and purification are needed by means of fractional precipitation, dialysis, ultrafiltration, electrophoresis and chromatography [47]. After years of research, the physiological and functional properties of rice bran polysaccharide have been gradually recognized, it has the functions of enhancing immune activity, anti-tumor, lowering blood sugar, lowering blood fat, anti-radiation, anti-oxidation and scavenging free radicals, so it is used as a functional food ingredient [48]. It is widely used in food, medicine and other industries with nutritional supplements. Japan is the most authoritative in the research of rice bran polysaccharides, and uses a variety of extraction processes to obtain a variety of rice bran polysaccharides. There are now rice bran polysaccharide products MGN-3 and NK 1000, etc. The former is an enzyme-modified rice bran polysaccharide, which the main component is arabian wood. The polysaccharide, which is a mixture of rice bran polysaccharide and fungal polysaccharide, can be used as a health food for enhancing immunity [49]. In addition, rice bran polysaccharide modification is also a new idea in recent years, because modification can produce new or improve the original physiological activity.

Table 1. Comprehensive utilization of rice and its by-products

\begin{tabular}{lll}
\hline By-products of rice & Main application & References \\
\hline Rice modified starch & New food for diabetic patients & {$[8,9,10]$} \\
$\quad$ Slowly digesting starch & Medium and low moisture foods & {$[1,12]$} \\
Resistant starch & Adsorption carrier for functional substances & {$[13,14,16]$} \\
$\quad$ Porous starch & To mimic the texture and mouthfeel of fat & {$[18,19]$} \\
Starch-based fat substitute & High-quality protein & {$[21,23,24,27]$} \\
Rice protein & Starch and high protein rice powder & {$[29,30,31]$} \\
Broken rice & & {$[38,39,40]$} \\
Rice bran & New health food oil & {$[42]$} \\
$\quad$ Rice bran oil & To lower blood fat and prevent cardiovascular diseases & {$[43,44]$} \\
Oryzanol & Biologically active functional substances & {$[45,46]$} \\
Rice bran wax & To develop nutritious and healthy foods & {$[47,48]$} \\
Rice bran nutrients & Nutrient fiber solid beverage & {$[49]$} \\
Rice nutrient fiber & Functional food ingredient & {$[50,51]$} \\
Rice bran polysaccharide & Functional food & {$[52]$} \\
Germinated brown rice & Convenient and cheap energy source & {$[53]$} \\
Rice husk & Raw material for carriers or composite materials & {$[54]$} \\
\end{tabular}




\section{Germinated Brown Rice}

About $60 \%$ to $70 \%$ of the physiologically active ingredients in rice are concentrated in rice bran, but since people usually consume white rice, most of the physiologically active ingredients in rice cannot enter the diet. Brown rice retains the rice bran rich in physiologically active ingredients, so its nutritional value is much higher than that of polished rice. However, brown rice has not been a staple food for human beings, and its nutrients have not been fully exploited. This is mainly because brown rice contains high crude fiber, enamel and waxy layers, which are blocked during cooking. Moisture enters the starch granules, and the swelling and water absorption are poor, resulting in higher gelatinization temperature of the starch, longer cooking time and difficulty in chewing [50]. At the same time, the brown rice contains astringency during the cooking process, and has a "slag feel" when consumed, and the digestion and absorption are also poor after ingestion.

The phytic acid contained in brown rice is antinutritional and easily combined with minerals such as calcium, iron and magnesium, thereby greatly reducing the bioavailability of minerals. After the brown rice is germinated, the starch is degraded into sugar, thus increasing the sweetness and flavor, solving the problem of unpleasantness of brown rice, and the physiological active ingredients in brown rice, such as dietary fiber, total phenolic acid, vitamins, reducing sugar, amino acids, trace amounts. Both elements and neurotransmitters are significantly increased before germination, especially neurotransmitters have blood pressure lowering, improved brain function, mental stability, promotion of long-term memory, promotion of growth hormone secretion, activation of renal function, activation of liver function, beauty, sobering, etc [51]. Therefore, improving the utilization value of brown rice through the germination process can not only improve the utilization efficiency of rice resources, but also improve the nutritional and dietary levels of people.

\section{Comprehensive Utilization of Rice Husk}

Rice husk is the largest by-product produced during the processing of rice, and its weight accounts for more than $20 \%$ of the total mass of rice. At present, a considerable part of China's rice husks have not been fully utilized, which not only pollutes the environment but also is an extreme waste of resources. In fact, rice husk is a resource with development potential. Its combustibles are more than $70 \%$, and its calorific value is $50 \%$ of standard coal. It is a convenient and cheap energy source. The carbonized rice husk is the production of water glass. The silicon in the rice hull is calcined under certain conditions to form porous amorphous silica particles, which has a large absorption surface activity and can be used as a raw material for various carriers or advanced composite materials [52].

In addition, since rice husk does not contain elements that poison single crystal silicon such as arsenic, fluorine, etc., it may be the best raw material for manufacturing solar cells. The rice husk can be made into a disposable, environmentally-friendly snack box that is safe, non-toxic, degradable, low in cost, and smooth in appearance, after being crushed, mixed, produced, formed, solidified, and surface-sprayed. This product has a large demand, low cost and broad market prospects. The rice husk can produce feed single-cell protein by secondary acid hydrolysis, and its by-product silica is an important industrial raw material [53]. The rice husk is made of synthetic resin as an adhesive. It can be made into package, furniture and other products by mixing and hot pressing to form rice husk. Rice husks can also be used as insulation agents for steel making, fiberboard, fillers for cultivating mushrooms, and the like. In addition, rice husks contain a variety of vitamins, enzymes and dietary fiber, which play an important role in promoting skin metabolism [54]. There are many unknown ingredients in rice husk, which have great development potential and broad application prospects.

\section{Outlook}

The research and development of intensive processing of rice is lagging behind, and the processing capacity is not strong. The vigorously developing and promoting the deep processing technology of rice and its by-products will extend the rice industry chain and greatly increasing the added value of rice, make full use of resources to supplement the shortage of high-quality edible oil, protein, starch and other products and make energy conservation and emission reduction, resource regeneration, turning waste into treasure. To revitalize the traditional rice processing industry, we must rely on colleges and universities with food science disciplines to vigorously cultivate new types of professionals, and give full play to the disciplinary advantages of scientific research institutes and strengthen the research on the application basis of rice food specialty. It is necessary for integration universities and research centers with enterprises, the establishment of rice food research and development center, and the formation of scientific and technological innovation and transformation of the results of the base. Moreover, we need to improve the technology center of large-scale rice circulation or processing enterprises to enhance the ability of enterprise innovation and professional institutions to promote new technologies, and actively promote the latest transformation of scientific research results. The full utilization of each rice by high-tech means to maximize the utilization value of rice resources can not only reduce environmental pollution, but also bring huge economic benefits.

\section{References}

[1] Qi W, Liang X, Yun T, Guo W. Growth and survival of microencapsulated probiotics prepared by emulsion and internal gelation. J Food Sci Technol. 2019; 56(3): 1398-404.

[2] Riaz B, Liang Q, Wan X, Wang K, Zhang C, Ye X. Folate content analysis of wheat cultivars developed in the North China Plain. Food Chem. 2019; 289: 377-83.

[3] Septiani P, Lanubile A, Stagnati L, Busconi M, Nelissen H, Pè $\mathrm{ME}$, et al. Unravelling the genetic basis of Fusarium seedling rot 
resistance in the MAGIC maize population: novel targets for breeding. Sci Rep. 2019; 9(1): 5665.

[4] Petri RM, Münnich M, Zebeli Q, Klevenhusen F. Graded replacement of corn grain with molassed sugar beet pulp modulates the fecal microbial community and hindgut fermentation profile in lactating dairy cows. J Dairy Sci. 2019; S0022-0302(19): 30298-X.

[5] Ding C, Liu Q, Li P. Distribution and quantitative analysis of phenolic compounds in fractions of Japonica and Indica rice. Food Chem. 2019; 274: 384-91.

[6] Riaz B, Liang Q, Wan X, Wang K, Zhang C, Ye X. Folate content analysis of wheat cultivars developed in the North China Plain. Food Chem. 2019; 289: 377-83.

[7] Septiani P, Lanubile A, Stagnati L, Busconi M, Nelissen H, Pè ME, et al. Unravelling the genetic basis of Fusarium seedling rot resistance in the MAGIC maize population: novel targets for breeding. Sci Rep. 2019; 9(1): 5665.

[8] Chen J, Li X, Chen L, Xie F. Starch film-coated microparticles for oral colon-specific drug delivery. Carbohydr Polym. 2018; 191: 242-54.

[9] Zhang C, Chen S, Ren X, Lu Y, Liu D, Cai X, et al. Molecular Structure and Physicochemical Properties of Starches from Rice with Different Amylose Contents Resulting from Modification of OsGBSSI Activity. J Agric Food Chem. 2017; 65(10): 2222-32.

[10] Mukamuhirwa A, Persson Hovmalm H, Bolinsson H, Ortiz R, Nyamangyoku O, Johansson E. Concurrent Drought and Temperature Stress in Rice-A Possible Result of the Predicted Climate Change: Effects on Yield Attributes, Eating Characteristics, and Health Promoting Compounds. Int J Environ Res Public Health. 2019; 16(6): E1043.

[11] Wang $\mathrm{H}, \mathrm{Wu} \mathrm{Y}$, Wang N, Yang L, Zhou Y. Effect of water content of high-amylose corn starch and glutinous rice starch combined with lipids on formation of starch-lipid complexes during deep-fat frying. Food Chem. 2019; 278: 515-22.

[12] Zeng F, Chen F, Kong F, Gao Q, Aadil RM, Yu S. Structure and digestibility of debranched and repeatedly crystallized waxy rice starch. Food Chem. 2015; 187: 348-53.

[13] Koh GY, Rowling MJ. Resistant starch as a novel dietary strategy to maintain kidney health in diabetes mellitus. Nutr Rev. 2017; 75(5): 350-60.

[14] Wei C, Xu B, Qin F, Yu H, Chen C, Meng X, et al. C-type starch from high-amylose rice resistant starch granules modified by antisense RNA inhibition of starch branching enzyme. J Agric Food Chem. 2010; 58(12): 7383-8.

[15] Sujka M, Pankiewicz U, Kowalski R, Nowosad K, NoszczykNowak A. Porous starch and its application in drug delivery systems. Polim Med. 2018; 48(1): 25-9.

[16] Tao H, Yan J, Zhao J, Tian Y, Jin Z, Xu X. Effect of multiple freezing/thawing cycles on the structural and functional properties of waxy rice starch. PLoS One. 2015; 10(5): e0127138.

[17] Srikaeo K, Laothongsan P, Lerdluksamee C. Effects of gums on physical properties, microstructure and starch digestibility of dried-natural fermented rice noodles. Int J Biol Macromol. 2018; 109: 517-23.

[18] Sheng Y, Xiao H, Guo C, Wu H, Wang X. Effects of exogenous gamma-aminobutyric acid on $\alpha$-amylase activity in the aleurone of barley seeds. Plant Physiol Biochem. 2018; 127: 39-46.

[19] Razzaq HA, Sutton KH, Motoi L. Altering the rate of glucose release from starch-based foods by spray-drying with an extract from barley. J Sci Food Agric. 2013; 93(11): 2654-9.

[20] Loubes MA, González LC, Tolaba MP. Pasting behaviour of high impact ball milled rice flours and its correlation with the starch structure. J Food Sci Technol. 2018; 55(8): 2985-93.

[21] Klu YA, Phillips RD, Chen J. Development of a Drinkable, Peanut-Based Dietary Supplement and Comparison of Its Nutritional and Microbiological Qualities with Commercial Products. J Food Sci. 2016; 81(5): H1309-12.

[22] Fujiwara Y, Aiki Y, Yang L, Takaiwa F, Kosaka A, Tsuji NM, et al. Extraction and purification of human interleukin-10 from transgenic rice seeds. Protein Expr Purif. 2010; 72(1): 125-30.

[23] De-Regil LM, Peña-Rosas JP, Laillou A, Moench-Pfanner R. Considerations for rice fortification in public health: conclusions of a technical consultation. Ann N Y Acad Sci. 2014; 1324: 1-6.

[24] Okuda M, Miyamoto M, Joyo M, Takahashi K, Goto-Yamamoto $\mathrm{N}$, Iida $\mathrm{S}$, et al. The relationship between rice protein composition and nitrogen compounds in sake. J Biosci Bioeng. 2016; 122(1): $70-8$.
[25] Joy JM, Lowery RP, Wilson JM, Purpura M, De Souza EO, Wilson SM, et al. The effects of 8 weeks of whey or rice protein supplementation on body composition and exercise performance. Nutr J. 2013; 12: 86.

[26] Arsa S, Theerakulkait C. Preparation, aroma characteristics and volatile compounds of flavorings from enzymatic hydrolyzed rice bran protein concentrate. J Sci Food Agric. 2018; 98(12): 4479-87.

[27] Sereewatthanawut I, Prapintip S, Watchiraruji K, Goto M, Sasaki M, Shotipruk A. Extraction of protein and amino acids from deoiled rice bran by subcritical water hydrolysis. Bioresour Technol. 2008; 99(3): 555-61.

[28] Liu H, Wan H, Xu S, Fang Z, Lin Y, Che L, et al. Influence of extrusion of corn and broken rice on energy content and growth performance of weaning pigs. Anim Sci J. 2016; 87(11): 1386-95.

[29] Nunes LV, de Barros Correa FF, de Oliva Neto P, Mayer CR, Escaramboni B, Campioni TS, et al. Lactic acid production from submerged fermentation of broken rice using undefined mixed culture. World J Microbiol Biotechnol. 2017; 33(4): 79.

[30] Setyawati YD, Ahsan SF, Ong LK, Soetaredjo FE, Ismadji S, Ju $\mathrm{YH}$. Production of glutinous rice flour from broken rice via ultrasonic assisted extraction of amylose. Food Chem. 2016; 203: 158-164.

[31] Wu P, Liao Z, Luo T, Chen L, Chen XD. Enhancement of Digestibility of Casein Powder and Raw Rice Particles in an Improved Dynamic Rat Stomach Model Through an Additional Rolling Mechanism. J Food Sci. 2017; 82(6): 1387-94.

[32] Park SY, Kim HY. Effect of Black Rice Powder Levels on Quality Properties of Emulsion-type Sausage. Korean J Food Sci Anim Resour. 2016; 36(6): 737-43.

[33] Park HY, Lee KW, Choi HD. Rice bran constituents: immunomodulatory and therapeutic activities. Food Funct. 2017; 8(3): 935-43.

[34] Sohail M, Rakha A, Butt MS, Iqbal MJ, Rashid S. Rice bran nutraceutics: A comprehensive review. Crit Rev Food Sci Nutr. 2017; 57(17): 3771-80.

[35] Ye B, Stary CM, Li X, Gao Q, Kang C, Xiong X. Engineering chimeric antigen receptor-T cells for cancer treatment. Mol Cancer 2018; $17(1): 32$.

[36] Fredman G, Spite M. Specialized pro-resolving mediators in cardiovascular diseases. Mol Aspects Med. 2017; 58: 65-71.

[37] Shao C, Yang F, Miao S, Liu W, Wang C, Shu Y, Shen H. Role of hypoxia-induced exosomes in tumor biology. Mol Cancer. 2018; 17(1): 120.

[38] Wallace TC, Murray R, Zelman KM. The Nutritional Value and Health Benefits of Chickpeas and Hummus. Nutrients. 2016; 8(12): E766.

[39] Wongwaiwech D, Weerawatanakorn M, Tharatha S, Ho CT. Comparative study on amount of nutraceuticals in by-products from solvent and cold pressing methods of rice bran oil processing J Food Drug Anal. 2019; 27(1): 71-82.

[40] Baschieri F, Cortelli P. Circadian rhythms of cardiovascular autonomic function: Physiology and clinical implications in neurodegenerative diseases. Auton Neurosci. 2019; 217: 91-101.

[41] Sookwong P, Mahatheeranont S. Supercritical CO2 Extraction of Rice Bran Oil -the Technology, Manufacture, and Applications. J Oleo Sci. 2017; 66(6): 557-564.

[42] Szcześniak KA, Ostaszewski P, Ciecierska A, Sadkowski T. Investigation of nutriactive phytochemical - gamma-oryzanol in experimental animal models. J Anim Physiol Anim Nutr (Berl). 2016; 100(4): 601-17.

[43] Cicero AF, Gaddi A. Rice bran oil and gamma-oryzanol in the treatment of hyperlipoproteinaemias and other conditions. Phytother Res. 2001; 15(4): 277-89.

[44] Doan CD, Tavernier I, Danthine S, Rimaux T, Dewettinck K. Physical compatibility between wax esters and triglycerides in hybrid shortenings and margarines prepared in rice bran oil. J Sci Food Agric. 2018; 98(3): 1042-51.

[45] Sharif MK, Butt MS, Anjum FM, Khan SH. Rice bran: a novel functional ingredient. Crit Rev Food Sci Nutr. 2014; 54(6): 807-16

[46] Pham T, Teoh KT, Savary BJ, Chen MH, McClung A, Lee SO. In Vitro Fermentation Patterns of Rice Bran Components by Human Gut Microbiota. Nutrients. 2017; 9(11): E1237.

[47] de Godoy MR, Kerr KR, Fahey GC Jr. Alternative dietary fiber sources in companion animal nutrition. Nutrients. 2013; 5(8): 3099-117. 
[48] Pang Y, Hou X, Yang C, Liu Y, Jiang G. Advances on chimeric antigen receptor-modified T-cell therapy for oncotherapy. Mol Cancer. 2018; 17(1): 91.

[49] Liu H, Lei C, He Q, Pan Z, Xiao D, Tao Y. Nuclear functions of mammalian MicroRNAs in gene regulation, immunity and cancer. Mol Cancer. 2018; 17(1): 64.

[50] Lee YT, Shim MJ, Goh HK, Mok C, Puligundla P. Effect of jet milling on the physicochemical properties, pasting properties, and in vitro starch digestibility of germinated brown rice flour. Food Chem. 2019; 282: 164-8.

[51] Thanoi S, Roboon J, Nudmamud-Thanoi S. Recovery effect of pre-germinated brown rice on the changes of sperm quality, testicular structure and androgen receptor expression in a rat model of drug addiction. Int J Med Sci. 2018; 15(9): 921-8.

[52] Bryant R, Proctor A, Hawkridge M, Jackson A, Yeater K, Counce P, Yan W, McClung A, Fjellstrom R. Genetic variation and association mapping of silica concentration in rice hulls using a germplasm collection. Genetica. 2011; 139(11-12): 1383-98.

[53] Shen Y. Rice Husk Silica-Derived Nanomaterials for Battery Applications: A Literature Review. J Agric Food Chem. 2017; 65(5): 995-1004.

[54] Sharif MK, Butt MS, Anjum FM, Khan SH. Rice bran: a novel functional ingredient. Crit Rev Food Sci Nutr. 2014; 54(6): 807-16.

(C) The Author(s) 2019. This article is an open access article distributed under the terms and conditions of the Creative Commons Attribution (CC BY) license (http://creativecommons.org/licenses/by/4.0/) 